$\underline{\text { Review Article }}$

\title{
COMPARISON OF EFFICACY AND SAFETY OF NEWER DRUGS APPROVED FOR THE TREATMENT OF MIGRAINE DISORDER: A REVIEW
}

\author{
PALANISAMY SIVANANDY ${ }^{1}$, AIDIL NATASHA ROSMAN ${ }^{2}$, AINAA DIYANA SHAPHERI ${ }^{2}$, TENG TENG CHEW ${ }^{2}$, \\ NATALIE JIA XIN LAU ${ }^{2}$, MICHELLE ZI YIN YEONG ${ }^{2}$, MICHELLA ONG², BIBI MUNIIRAH UMMEH HAANI MEETOOA ${ }^{2}$, \\ ELAINE NG ${ }^{2}$, KARTHIKAYINI KRISHNASAMY ${ }^{3}$, ARULKUMARAN GOVINDARAJAN ${ }^{4}$, AKILA \\ SHANMUGASUNDARAM ${ }^{5}$
}

1Department of Pharmacy Practice, International Medical University, Kuala Lumpur 57000, Malaysia, ${ }^{2}$ School of Pharmacy, International Medical University, Kuala Lumpur 57000, Malaysia, ${ }^{3}$ Department of Nursing, University Malaya Medical Centre, Kuala Lumpur 59100, Malaysia, ${ }^{4}$ Department of Pharmaceutics, KMCH College of Pharmacy, Kovai Estate, Kalapatti, Coimbatore 641048, Tamil Nadu, India, ${ }^{5}$ Assistant Professor, Department of Physical Education, Bharathiar University, Coimbatore 641046, Tamil Nadu, India Email: sivapalanisamy@yahoo.co.in

Received: 13 Mar 2020, Revised and Accepted: 21 May 2020

\section{ABSTRACT}

Migraine is a recurrent throbbing or pulsing headache with moderate to severe pain intensity. The pain is often one side of the head with nausea and weakness symptoms. Around 12 percent of Americans, 9 percent of Asians experiences migraine and the prevalence is highest among South Koreans $(22.3 \%)$. The outcome of chronic migraine treatment can be quite disheartening, causing patients to feel out of options who have tried multiple treatments with no results. Poor efficacy, tolerability and safety of migraine preventive therapy in clinical practice lead to poor compliance and failure of therapy. The mean change in number or frequency of headache is considered as the outcome measure of migraine prevention therapy. Upon comparing all migraine prevention therapy, the Fremanezumab, Eptinezumab, Galcanezumab and Erenumab were considered as the front runner in controlling the severity and frequency of migraine. Among these drugs, Erenumab was most effective in controlling the frequency of migraine episodes as it produces more than 50 percent reduction in the mean number of monthly migraine days (MMD) over week 9-week 12. In addition to drug therapy, adequate rest, balanced diet, yoga and meditation will help patients to get rid of migraine severity. A multi-dimensional approach is essential for better control over migraine symptoms.

Keywords: Headache, Migraineurs, Nausea, Safety, Tolerability, Phonophobia

(C) 2020 The Authors. Published by Innovare Academic Sciences Pvt Ltd. This is an open access article under the CC BY license (http://creativecommons.org/licenses/by/4.0/) DOI: http://dx.doi.org/10.22159/ijpps.2020v12i7.37440. Journal homepage: https://innovareacademics.in/journals/index.php/ijpps

\section{INTRODUCTION}

Migraine is an extremely usual, persistent, and normally geneticallyrelated neurovascular disorder which occurs at irregular intervals [1]. It is a weakening brain disorder impacting approximately fifteen percent of the world population. Generally, migraine attacks comprise of severe headaches which accompany by a group of symptoms, lasting for four to seventy-two hours, for instance, nausea, vomiting, photo-and phonophobia [2]. As a major cause of neurological disability worldwide and due to its nature, it is undoubtedly having a significant effect on society [3-5]. In addition, migraine can be categorized into episodic migraine and chronic migraine. The most common form of migraine is episodic migraine, has an attack of headache happening for less than $15 \mathrm{~d}$ monthly [3]. As a multifactorial genetic disorder, migraine has two mechanisms, which are the neuronal and vascular pathway that includes several dozens of gene variants with minimal effect size [4]. There are around twenty to thirty percent of migraineurs are affected by short-term focal neurologic symptoms, which can occur before or during the headache and it is called aura [5]. The frequency, duration, and intensity of the migraine attack can be different among individuals. The occurrence of temporary disability due to migraine attack creates a significant impact to the migraine patients' work and activities lead to impairment in productivity and quality of life of the patients [1]

The data extracted for this review is mainly on the antimigraine drugs used in the treatment of various migraine disorders. The main source of data used is PubMed, Nvivo, Mendeley, Evernote, CiteUlike, Biohunter, Delvehealth, Scicurve, and Google Scholar, etc. Articles on complementary therapy on migraine disorder and animal studies were excluded. The antimigraine drugs included in our studies are those that were approved by US-FDA, as according to Centre Watch. All the authors independently extracted the relevant information from studies that fulfilled our inclusion criteria and any disagreements were resolved with consensus. The information extracted included the trial phase, region, conditions of subjects and the outcome measures. This information was gathered and summarized into paragraphs, introducing each antimigraine drug comprehensively.

\section{Epidemiology}

As a neurovascular disease, migraine is currently being considered as a severe and prevalent health issue. To be more precise, it has become the sixth-leading cause disability globally and the third-leading cause of disability in people of age less than 50-yearold [6-9].

Migraine has affected different populations, with the highest incidence in Europe and North America (13\%), followed by Asia (9\%) [10]. Besides, it has been shown through a recent study regarding the headache disorders in India, which outlined individuals suffering from various headaches, of which $26 \%$ of them suffer from migraine [11]. Furthermore, the 2010 Global Burden of Disease Study had presented that the worldwide prevalence of migraine was $14.7 \%$, which was slightly lower as compared to the incidence of tension-type headache (20.1\%) [12-14]. In 2013, the same study was being conducted and revealed that neurological disorders had contributed to over half of all years lost to disability [10-16]. In 2015, the study reported that migraine was considered one of the eight chronic diseases which influenced more than $10 \%$ of the global population [17]. Gender wise, it had a greater impact on women compared to men, with prevalence of $17 \%$ and $6 \%$ respectively, resulting to a remarkable socioeconomic burden to the society. Migraine was then proved to be the second-highest cause of years lived with disability globally in the 2016 Global Burden of Disease study [18] 
Migraine also related to the people's socioeconomic burden, with respect to both standard of living and lost efficacy [19]. This is supported by previous studies, which indicated that about 9 out of 10 migraine patients are functionally affected during an attack, approximately half of them are gravely impaired and in need of bed rest. It has also been reported that those with migraine are only about half as productive at work compared to those without $[8,20]$. Furthermore, the burden of migraine is higher in part-timers or those who are jobless, has low socioeconomic status, and no government insurance. These populations are presumably to have limited access to health care and treatment for their headaches. In addition, these people are more likely to be exposed to triggers and other factors that can aggravate headache. Therefore, this is progressively relevant as the managements of migraine and other severe headaches move from symptom-based, non-specific therapies to more specific, individualized, and cost-effective treatments such as the new anti-calcitonin gene-related peptide (anti-CGRP) antibodies. It is crucial to understand the distribution of headache in specific segments of the population as this allows the treatments to be accessible to those most in need [21]. The current conventional drugs control the severity of migraine at a certain level; however, no complete salvage from the recurrent migraine attacks. A novel antimigraine therapy is needed to control the severity and recurrent attacks, and also has the least side-effects. Hence, a review was carried out to compare the mechanism, efficacy and safety of antimigraine drugs that indicated for the treatment of migraine disorder.

\begin{abstract}
Management
Migraine is generally managed with a different class of drugs, namely non-steroidal anti-inflammatory drugs (NSAID), 5-hydroxy tryptamine (5HT)-agonists, ergot preparations, and specific drugs targeting the receptors. Prophylactic treatment choices for migraines include drugs developed for diseases other than migraines such as depression, epilepsy and hypertension [22]. In the past ten years, inhibiting CGRP has appeared to be a possible mechanism to prevent migraine attacks. This is supported by recent evidence suggesting that dysfunctional activation of the trigeminovascular system involving CGRP is implied in migraine pathogenesis [22-25]. The drugs which are commonly used in migraine are discussed comprehensively below emphasizing their mechanism of action, efficacy and safety in migraine prevention or control. The summary of the efficacy and safety of newer drugs that recently approved for the treatment of migraine are compared and presented in table 1 .
\end{abstract}

Table 1: Comparison of efficacy and safety of newer drugs that approved by US-FDA for the treatment of migraine disorder

\begin{tabular}{ll}
\hline Author name & \multicolumn{1}{c}{ Title of the article } \\
\hline CGRP antagonist \\
\hline $\begin{array}{ll}\text { 1. Fremanezumab } \\
\text { Dodick } \text { et al., }\end{array}$ & $\begin{array}{l}\text { Effect of fremanezumab } \\
\text { compared with placebo } \\
\text { for prevention of episodic } \\
\text { migraine: A randomized } \\
\text { clinical trial }\end{array}$ \\
& \\
Bigal et al., & $\begin{array}{l}\text { Safety, tolerability, and } \\
\text { [50] }\end{array}$ \\
& $\begin{array}{l}\text { preventive treatment of } \\
\text { chronic migraine: A } \\
\text { multicentre, randomised, } \\
\text { double-blind, placebo- }\end{array}$ \\
& $\begin{array}{l}\text { controlled, phase } 2 \mathrm{~b} \text { study } \\
\text { Fremanezumab as an add- }\end{array}$ \\
& $\begin{array}{l}\text { on treatment for patients } \\
\text { t51] }\end{array}$ \\
& $\begin{array}{l}\text { treated with other } \\
\text { migraine preventive } \\
\text { medicines }\end{array}$
\end{tabular}

\section{Eptinezumab}

Dodick et al., $\quad$ Safety and efficacy of ALD [52] 403 for the prevention of frequent episodic migraine: a randomised, double-blind, placebocontrolled, exploratory phase 2 trial

Multicentre, randomised, double-blind, double-dummy, placebo-controlled, parallel-group phase 2b study Randomized Placebo-controlled studies

andomized, double-blinded, placebo-controlled, phase 3 study

Randomised, double blind, placebocontrolled,

exploratory, proofof-concept phase 2 trial of an intravenous dose of ALD 403 at 26 centres in the USA

Single-dose, placebo-controlled study, exploratory phase 2 trial

\section{Mean change}

from baseline in

the mean number of monthly migraine days during the 12week period after the first dose

Mean change in the number of headache-hours

$$
\begin{aligned}
& \text { 675/225 mg group: } \\
& -59 \cdot 84 \mathrm{~h} \\
& 900 \mathrm{mg} \\
& \text { group: }-67 \cdot 51 \mathrm{~h}
\end{aligned}
$$

Mean change in migraine days migraine days Frequent
migraine
episodes

1.3-to 1.5-day reduction in the mean number of monthly migraine days over a 12 week period Safety

-

Injection site-related pain

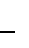


3. Galcanezumab

Forderreuther Preventive effects of et al., [58] galcanezumab in adult patients with episodic or chronic migraine are persistent: data from the phase 3 , randomized double-blind, placebocontrolled EVOLVE-1, EVOLVE-2, and REGAIN studies

Skljarevski et $\quad$ Efficacy and safety of al., [57] galcanezumab for the prevention of episodic migraine: Results of the EVOLVE-2 Phase 3 randomized controlled clinical trial

Skljarevski et $\quad$ Effect of Different Doses of al., [68] Galcanezumab vs Placebo

for Episodic Migraine Prevention A Randomized Clinical Trial

\section{CGRP-Receptor Antagonist}

\section{Erenumab}

Dodick et al. [59]

\section{ARISE: A Phase 3 randomized trial of erenumab for episodic migraine}

Reuter et al.,

Efficacy and tolerability of erenumab in patients with episodic migraine in whom two-to-four previous preventive treatments were unsuccessful: a randomized, double-blind, placebo-controlled, phase 3b study (LIBERTY)

Goadsby et al., A Controlled Trial of [61] Erenumab for Episodic Migraine (STRIVE)
Randomized, double-blinded, placebo-controlled, phase 3 study

Randomized, double-blinded, placebo-controlled multicenter, phase 3 study at 109 study centres in 11 countries

Randomized, double-blinded, placebo-controlled, phase $2 b$ study in clinics of 37 licensed physicians with a specialty

Randomized, multicenter, doubleblind, placebocontrolled, phase 3 study

Criteria: 577 participants with episodic migraine (EM), had 4-15 MMD with or without aura for at least 12 mo before the study Randomized, multicenter, doubleblind, placebocontrolled, phase $3 \mathrm{~b}$ study

Criteria: 246

participants with a history of EM with or without aura for at least $12 \mathrm{mo}$, had migraine for average of 4-14d per months over 3 mo before the screening, had unsuccessful treatment with between two-tofour preventive treatments.

Randomized, multicenter, doubleblind, placebocontrolled, phase 3 study
Mean monthly migraine headache days (MHDs)

Mean monthly migraine

headache days (MHDs)

Frequency of migraine

headache days (MHDs)

Change in monthly migraine days (MMD) over Month 3 of study.

$\geq 50 \%$ reduction in the mean number of MMD over Week 9Week 12.

Change in the mean number of MMD over Month 4-Month 6
At month 1, 20\% of patients had a sustained response of $\geq 50 \%$ reduction of MHDs over 6 months; about $41 \%$ of patients maintained $\geq 50 \%$ response over $\geq 3$ months

120 mg: -4.3 MHDs 240 mg: -4.2 MHDs

120 mg: -4.8 MHDs

70 mg SC monthly vs placebo $(\mathrm{p}<0.001)$

-2.9 d change in MMD from baseline

$40 \mathrm{mg}$ (via two divided $70 \mathrm{mg}$ injections) SC monthly vs placebo erenumab group had $\geq 50 \%$ reduction in mean number of MMD vs $17 / 124$ of placebo group had $\geq 50 \%$ reduction in mean number of MMD $36 / 119$ of

back pain ( 4 vs 3 ), arthralgia (4 vs 1 ), and nausea (2 vs 3 ) No infusion reactions were reported 2 patients in the eptinezumab group and 1 patient in the placebo group experience serious adverse events.

Injection site-related pain

Injection site-related pain

Injection site-related pain

Most common AE-Upper respiratory tract infection

Most common AE-pain at the injection site

70 mg SC monthly, 140 mg SC monthly vs placebo $(\mathrm{p}<0.001$ for each dose vs placebo)

$70 \mathrm{mg}$ shows-3.2 d
Most common AE-

Nasopharyngitis 


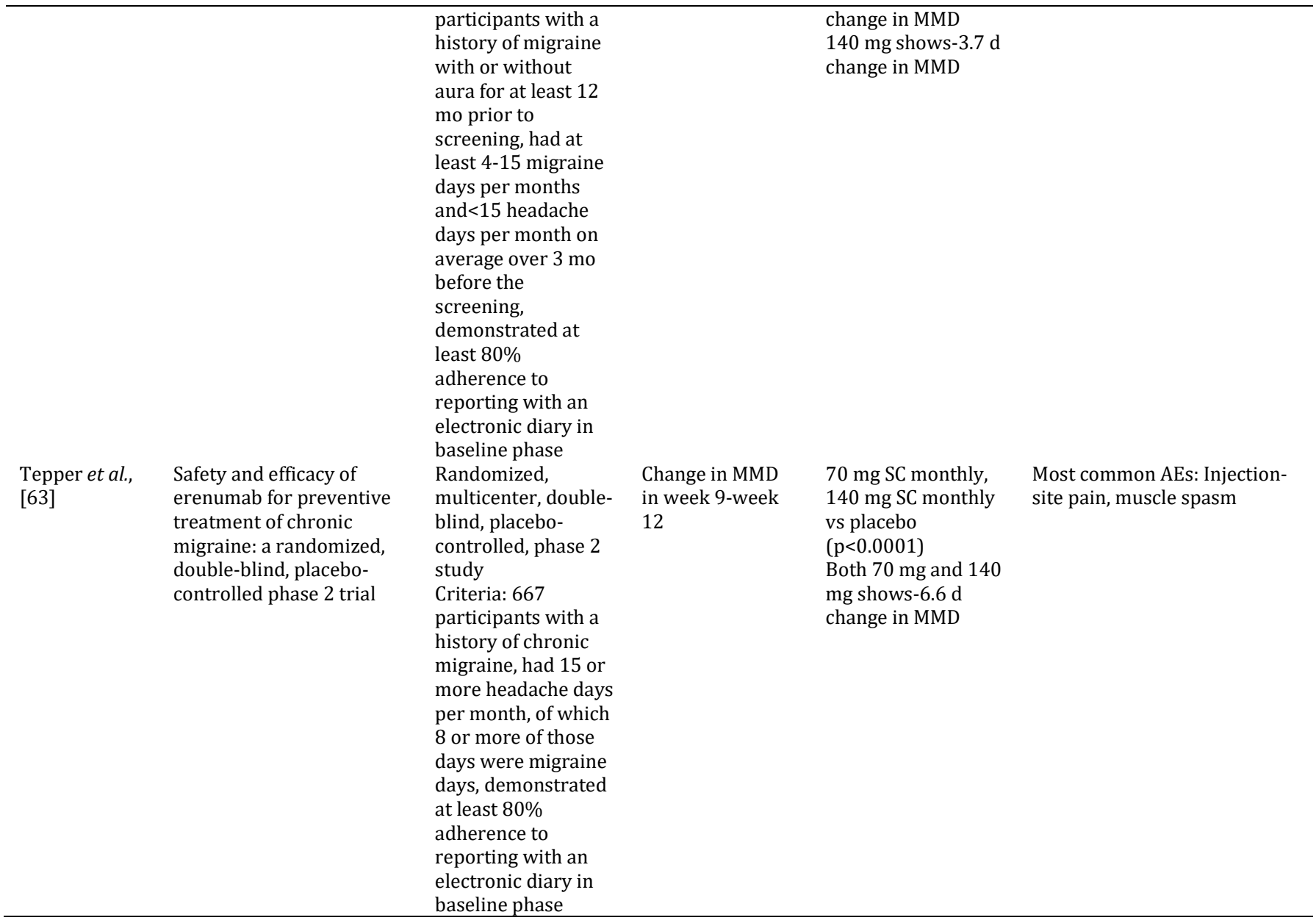

\section{Mechanism of action}

\section{a) Non-steroidal anti-inflammatory drugs}

Acetaminophen and NSAIDs which possess analgesic and antiinflammatory actions in migraine by inhibiting the enzyme cyclooxygenase (COX) to reduce prostaglandin synthesis from arachidonic acid [26]. There are two cyclooxygenase enzymes which are COX-1 is widely expressed in gastrointestinal tract, whereas COX-2 is widely predominated at sites of inflammation [27]. Aspirin inactivates COX-1 irreversibly and inhibit the production of prostaglandin $\left(\mathrm{PGH}_{2}\right)$ where it acts as a primary precursor of thromboxane $A_{2}$. Aspirin interacts with the amino acid Arg120 which result obstructing of the accessibility of arachidonic acid to the Tyr385 hydrophobic channel at catalytic site [28].

\section{b) 5 -hydroxy tryptamine (5HT)-agonist}

In the $1990 \mathrm{~s}$, the emergence of the selective $5-\mathrm{HT}_{1 \mathrm{~B}}$ and $5-\mathrm{HT}_{1 \mathrm{D}}$ receptors agonists was a significant advancement for the acute management of migraine. Triptans exhibit antimigraine effects through cranial vasoconstriction and by inhibition of CGRP in the perivascular nerve terminals, subsequently reducing the activation of trigeminal nociceptors [29-31]. A few examples of the triptans include zolmitriptan, rizatriptan, and naratriptan.

\section{c) Ergots}

According to the vascular theories of migraine, the ergot alkaloids as vasoconstrictors were turned into one of the earliest approaches towards migraine attacks [32]. Antimigraine drugs introduced to the market were ergotamine (E) tartrate as the first pure ergot alkaloid and dihydroergotamine (DHE) [33]. Ergots are indicated for migraines that also present with a long period and infrequent headaches and to patients who are likely to adhere with dosing restrictions. E and DHE once remained as the only available acute specific antimigraine treatments until sumatriptans were developed in 1980s. The ergots have high selectivity for various receptors, such as dopamine, noradrenaline and serotonin (5-hydroxytryptamine). E and DHE interact with 5-HT1A, 1B, 1D, 1F, 2A, 2C, 3, 4 subtypes.

\section{d) Others drugs}

Botulinum toxin A (BoNT-A), due to its healing properties and its ability to alleviate pain, an increasing number of studies have been carried out for the past ten years to investigate the efficiency of BoNT-A in treating migraines. Animal and human studies have revealed that BoNT-A inhibits the release of the neurotransmitters glutamate A, calcitonin gene-related peptide and Substance-P, which are important mediators of inflammatory pain. Hence, nociceptive signals reaching the central system are minimized. BoNT-A is administered peripherally in the form of injections to the head or neck [32].

\section{Specific management}

The specific management of migraine includes CGRP antagonists and its receptor antagonists, both are considered simultaneously in some cases depends on the severity of the condition.

\section{Calcitonin gene-related peptide antagonists}

\section{a) Fremanezumab}

Fremanezumab, also known as Ajovy is the second drug after erenumab (Aimovig) to be approved by the FDA for the preventive treatment of migraines. Engineered by recombinant DNA technology, Fremanezumab is a fully-humanized monoclonal antibody. It has a strong affinity for CGRP ligand, a neuropeptide that is strongly implicated in migraine pathophysiology. This antibody is made up of 1324 amino acids and has a molecular weight of approximately $148 \mathrm{kDa}$. Being highly specific, tolerable and safe, 
Fremanezumab has proved to be an ideal drug development for migraines. Goadsby et al., found direct evidence that the prophylactic effect of CGRP-mAbs is achieved mainly through their ability to prevent the activation of peripheral trigeminovascular neurons of the A $\delta$ type by events that lead to cerebral release of CGRP during a migraine headache $[34,35]$. While erenumab blocks CGRP receptor, fremanezumab binds to the CGRP molecule and blocks its attachment to the CGRP receptor. Fremanezumab has an estimated half-life of approximately $31 \mathrm{~d}$. Two subcutaneous dosing options of Ajovy exist which are a monthly dose of $225 \mathrm{mg}$ or 675 $\mathrm{mg}$ to be administered every $3 \mathrm{mo}$. In clinical trials, hypersensitivity reactions including rash and pruritus were reported at injection sites within hours to one month after administration.

\section{b) Eptinezumab}

Eptinezumab, ALD403 is a fully-humanized IgG1 antibody that binds specifically and selectively to both alpha and beta forms of the human CGRP. ALD403 also binds potently $(\mathrm{Kd}<20 \mathrm{pM})$ to human CGRP [36].

\section{c) Galcanezumab}

One of the recently approved drugs for migraine prevention called galcanezumab-gnlm, also known as LY2951742, is an entirely humanized monoclonal antibody which potently and selectively binds to CGRP ligand and blocks its binding to the receptor, hindering CGRP-mediated vasodilation effects $[36,37]$.

\section{Calcitonin gene-related peptide receptor antagonists}

\section{a) Erenumab}

Erenumab is the first FDA-approved GCRP-receptor monoclonal antibody specifically developed for the management of migraines [38]. It is formerly known as AMG334, due to its nature as a fully human monoclonal antibody, it specifically attaches to CGRP receptor. Attachment sites of this receptor is closely related to receptor activity-modifying protein 1 (RAMP1) complex and calcitonin receptor-like receptor (CLR). Through this binding, the biological activities of CGRP are blocked with an IC50 $(2.3 \pm 0.9 \mathrm{nM})$ [39]. Erenumab is 5000 -fold more specific for CGRP receptor as compared to any other human calcitonin family receptors. Erenumab is considered as a very large molecule where its molecular weight is about $150.000 \mathrm{kDA}$. In the contrary, small molecule of CGRP receptor antagonists have molecular weight of less than $500 \mathrm{kDA}$, making it possible to enter the central nervous system (CNS) [40]. Due to its large molecular size, it poses a low risk of penetration into the blood-brain barrier (BBB) that can result in adverse reactions associated with CNS. According to Eftekhari et al., [41] erenumab has mode of action outside of BBB, specifically at trigeminal ganglion. Site of expression of CGRP are in neurons of greater sizes, specifically $A \delta$ neurons as well as the cells of satellite glial. Meanwhile, CGRP receptors are dispersed in c-fiber neurons of relatively smaller diameter. Inhibitory action of erenumab on activation of $A \delta$ results in the preventive effect in migraines. Erenumab possess half-life of $26 \mathrm{~d}$, and this explains the need for drug administration to be done only once a month [42]. Route of administration of this drug is through subcutaneous injection thus having its primary metabolism handled by the reticuloendothelial system. It is found that erenumab is not eliminated via hepatic, renal or biliary process, which lowers the risk of drug-drug interactions by not competing with other drugs via these excretion pathways. Through various studies, erenumab is considered to be highly potent in inhibiting the capsaicin-induced dermal blood flow (CIDBF) [38].

\section{Efficacy}

The efficacy of recently marketed antimigraine drugs was critically analyzed using the reduction in pain intensity and the number of headache-free days. The details are presented below.

\section{a) Non-steroidal anti-inflammatory drugs}

Aspirin is well-known in the treatment of migraine. A systematic Cochrane review discovered that a single dose of $1 \mathrm{~g}$ of aspirin relieves headache in $52 \%$ of attacks and $32 \%$ for placebo at $2 \mathrm{~h}$, whereas $24 \%$ shown free of pain at $2 \mathrm{~h}$ compared to $11 \%$ for placebo. At a dose of $1 \mathrm{~g}$ acetaminophen alone had high efficiency while at a dose of $650 \mathrm{mg}$, acetaminophen was not better than placebo [27]. Acetaminophen, other NSAIDs and aspirin are the most widely used drugs for migraine attack. Nonetheless, many randomized controlled trails proved that the efficacy of acetaminophen is slightly lower than other NSAIDs for a migraine attack.

\section{b) 5-hydroxy tryptamine (5HT)-agonists}

Oral sumatriptan $50 \mathrm{mg}$ and eletriptan $40 \mathrm{mg}$ are the most advantageous as a first-line specific acute migraine therapy, while subcutaneous sumatriptan $6 \mathrm{mg}$ is the most effective currently marketed drug [43]. Zolmitriptan has an efficacy of $62 \%$ at $2 \mathrm{~h}$ and up to $78 \%$ within $4 \mathrm{~h}$ on a regular dose 2.5 to $5 \mathrm{mg}$ orally or as intranasal spray. One of the new delivery methods for an aged acute migraine therapy is Zecuity ${ }^{\circledR}$ which is a battery-powered, transdermal sumatriptan patch considered more suitable for migraine headaches and cluster headaches [44].

\section{c) Ergots}

Oral formulations of ergot are poorly absorbed due to extensive first-pass metabolism with nausea as its main side effect, while its rectal form shows higher efficacy where relatively higher plasma levels are observed. Rectal formulation of ergot is thus recommended for patients with early onset of migraine with severe nausea and vomiting. DHE are currently available as intravenous, intramuscular, subcutaneous and intranasal formulations. Among ergot alkaloids, DHE is at an advantage as it is marketed with various administration possibilities, is relatively a weaker vasoconstrictor [45] and has longer half-life. Due to its longer half-life, it has a low risk of medication overuse [46] as well as lesser side effects. Usage of ergots as antimigraine should be limited only to younger patients who respond poorly to other treatments [47].

\section{d) Others drugs}

Similar to other preventive migraine treatments, it has been found that the advantageous effects of BoNT-A could be noticed mostly in $2^{\text {nd }}$ and $3^{\text {rd }}$ months of post-treatment period. This is in accordance with findings which state that it takes up to $3 \mathrm{w}$ for botulinum toxin to achieve its maximum efficiency. In patients suffering from chronic migraine, it can be noted that BoNT-A reduces the number of migraine days by 2 d over a period of one month. Due to the unavailability of high-quality evidence, it remains unclear as to whether BoNT-A is effective in preventing episodic migraine [48].

\section{Specific management}

\section{Calcitonin gene-related peptide antagonists}

\section{a) Fremanezumab}

Dodick et al., enrolled 875 participants in a phase 3, double-blind, placebo-controlled, parallel group study whereby fremanezumab was administered either monthly or a higher dose was given only once while others received placebo. The primary end point being investigated in this study was the mean change from baseline in the mean number of MMD, $12 \mathrm{w}$ after the first injection. Based on the findings, $12 \mathrm{w}$ after receiving the first dose, a reduction from 8.9 to 4.9 MMD was observed for the monthly fremanezumab dosing group. Patients received a single higher dose of fremanezumab showed a 9.2 to 5.3 MMD reduction while placebo group showed a decrease from 9.1 to $6.5 \mathrm{~d}$. The MMD declined by at least half in $47.7 \%$ of patients who were injected with fremanezumab monthly and $44.4 \%$ those who received the single higher dose of fremanezumab as compared with $27.9 \%$ for the placebo group. This study also concluded that among patients with episodic migraine, subcutaneous fremanezumab reduced the MMD by 1.3 to $1.5 \mathrm{~d}$ [49]. In another phase $2 \mathrm{~b}$, double-blind, double-dummy, placebo-controlled, parallel-group study conducted by Bigal et al., participants were enrolled to receive $675 / 225 \mathrm{mg}$ fremanezumab, $900 \mathrm{mg}$ fremanezumab or placebo. During weeks 912 , findings showed that in the $675 / 225 \mathrm{mg}$ group, the mean change from baseline in the number of headache-hours was -59.84 while in the $900 \mathrm{mg}$ group, the change was $-67.51 \mathrm{~h}$ and $-37.10 \mathrm{~h}$ in the placebo group. A 38\% decrease in the headache-hours was observed for those who received $675 / 225 \mathrm{mg}$ dose of fremanezumab, while in 
the $900 \mathrm{mg}$ group, headache-hours decreased by $43 \%$ compared to only $22 \%$ in the placebo group [50]. In two randomized placebocontrolled studies carried by Cohen et al., the total decline in migraine days was 12.4 for fremanezumab and 7.4 for placebo during the study period, in patients who were already on other migraine preventive medications. Decreases in moderate/severe headache days were also observed. Similarly, the number of days where acute medication was used for headaches decreased compared to placebo. The study concluded that in patients who were already on anti-migraine therapy, fremanezumab significantly reduced the MMD as well as moderate to severe headache days, and days whereby acute medication was used. Hence, the efficacy of fremanezumab as a complementary therapy to other migraine preventive medications was hence validated by this study [51].

\section{b) Eptinezumab}

Dodick et al., in their randomized, double-blind, placebo-controlled, exploratory phase 2 trial in migraine patient population stated due to its momentary and mild or moderate-severe adverse effects, Eptinezumab (ALD403) was normally safe and well-tolerated. On week 5-8, the average number of days with migraine reduced compared to initial number. In addition, $75 \%$ of the patients treated with ALD403 experienced a decrease of $50 \%$ of migraine days, whereas another $44 \%$ undergone a decrease of $75 \%$ at this same time point. Moreover, $16 \%$ of the patients in ALD403 indicated in a posthoc analysis do not have any migraine attacks in which there's a $100 \%$ decline in day of migraine in the entire study period of twelve weeks. Nonetheless, placebo group do not show fully decline in migraine days if compared to treatment group [52]. Dodick et al., [53] in a single-dose and placebo-controlled study demonstrated patients with frequent migraine attacks received single dose of eptinezumab by intravenous route; where 163 participants aged between 18 and $55 \mathrm{y}$ old with 5 to 14 migraine were randomly assigned to receive either $1 \mathrm{gm}$ eptinezumab or placebo intravenously every $28 \mathrm{~d}$ for up to $24 \mathrm{w}$. In which, $57 \%$ of the patients from the treatment group experienced mild to moderate adverse effects compared to placebo group. Generally, the adverse effects were arthralgia, nausea, upper respiratory tract infections, fatigue, urinary tract infections, back pain.

Seven patients from the treatment group and 6 patients from placebo experienced upper respiratory tract infections; whereas only 1 patient from ALD403 group and 4 patients from placebo had urinary tract infections and arthralgia. There is an equal number $(n=3)$ of patients from both group noted with from fatigue, 4 and 2 patients experienced back pain and nausea respectively. There were 2 patients from ALD403 and 1 patient from placebo group experienced serious adverse effects. It is undeniable that higher response rates showed in ALD403 group with approximately $20 \%$ higher than placebo. Furthermore, $16 \%$ of patients were reported to have no migraine days when treated with eptinezumab [54].

\section{c) Galcanezumab}

Schuster et al., in their phase two randomized, controlled trial involving 218 participants with episodic migraine, each participant received a subcutaneous $150 \mathrm{mg}$ dose of galcanezumab or a placebo every fortnight [55]. The primary endpoint of reduction in monthly migraine headache days (MHDs) was achieved during the third month of therapy with a monthly decrease of 4.2 and 3.0 MHDs in the treatment and placebo group, respectively. The $100 \%$ responder rate, defined as absence of migraine attacks during the 3-month trial, was also lower in the controlled group than in the treatment group [55]. A study by Camporeale et al., compared the efficacy of $120 \mathrm{mg}$ and $240 \mathrm{mg}$ of galcanezumab, and reported that the overall mean reduction in MHDs over $12 \mathrm{mo}$ were 5.6 for $120 \mathrm{mg}$ and 6.5 for $240 \mathrm{mg}$. Additionally, the improved functioning level was observed, and headache-related dysfunction was reduced in both dose groups [56]. Subsequently, Skjarevski et al., in their randomized, double-blinded, placebo-controlled, multicenter, phase 3 study at 109 centers in 11 countries found a reduced mean monthly MHDs of 4.3 and 4.2 for $120 \mathrm{mg}$ and $240 \mathrm{mg}$ of galcanezumab, respectively [57]. The most recent finding was from a phase 3 study conducted by Forderreuther et al., whereby $20 \%$ of the patients had a sustained response of equal or more than $50 \%$ reduction of MHDs over six months. Among the $20 \%, 41 \%$ of them maintained the said response for three months or more [58].

\section{Calcitonin gene-related peptide receptor antagonists}

\section{a) Erenumab}

ARISE [59] was a phase 3 study conducted over 3 mo, in which the monthly subcutaneous injections of $70 \mathrm{mg}$ of erenumab vs placebo were studied in 577 episodic migraine (EM) patients, and the change in MMD as primary outcome was assessed in month 3 of the treatment phase. In regards to this end-point, erenumab showed more promising results relative to placebo where it showed-2.9 d change of MMD from its baseline while placebo group showed1.8days change of MMD. This further supports an earlier consideration that $70 \mathrm{mg}$ is the minimal effective dose in patients with EM [60]. In STRIVE [61], of the same study design as the previous trial, $70 \mathrm{mg}$ and $140 \mathrm{mg}$ of erenumab were used. Results showed a reduction in MMD of $3.0 \mathrm{~d}$ in patients with $70 \mathrm{mg}$, and 3.5 d' reduction with $140 \mathrm{mg}$, whereas $1.7 \mathrm{~d}$ ' reduction in MMD was observed in placebo group. Erenumab at both doses elicited a change in MMD that was significantly higher by almost $2 \mathrm{~d}$ compared to placebo. The efficacy of $140 \mathrm{mg}$ Erenumab was higher compared to $70 \mathrm{mg}$ and placebo regarding all endpoints. In another phase $3 \mathrm{~b}$ study LIBERTY [62], patients whose previous preventive treatments were unsuccessful in EM, and administered with either placebo or $140 \mathrm{mg}$ of erenumab given in two subcutaneous injections of 70 $\mathrm{mg} / 1 \mathrm{ml}$. At week 12, among 119 patients who received erenumab, $30 \%$ of them showed $\geq 50 \%$ decline in the mean number of MMD. Meanwhile, in placebo group consisting of 124 patients, only $14 \%$ showed the same result. Additionally, through weeks 0-4 and weeks $5-8$, relative to placebo group, higher proportion of the erenumab group had $\geq 50 \%$ decrease in mean number of MMD. For secondary endpoints, erenumab group showed a reduced MMD specifically by $1.8 \mathrm{~d}$. while placebo reduced $0.2 \mathrm{~d}$ in MMD. This further proves erenumab as an alternative therapeutic agent in EM patients whom other traditional preventive treatments are contraindicated, unsuccessful or poorly tolerated.

In addition to that, another phase 2 trial [63] demonstrated the efficacy of treatment with erenumab given in 667 patients suffering from chronic migraines. Patients were assigned with either monthly subcutaneous placebo, $70 \mathrm{mg}$ or $140 \mathrm{mg}$ of erenumab. Patients receiving $70 \mathrm{mg}$ or $140 \mathrm{mg}$ of erenumab demonstrated a significant change in MMD of-6.6days for both dose vs placebo at-4.2days. Besides, $40 \%$ of a group of 188 patients treated with $70 \mathrm{mg}$ erenumab and $41 \%$ of 187 patients given $140 \mathrm{mg}$ erenumab obtained $\geq 50 \%$ reduction in mean number of MMD as compared to $23 \%$ of 281 patients in placebo. Erenumab shows promising efficacy in prevention of both chronic as well as EM through various demonstrations in both phase 2 and 3 trials.

\section{Safety}

The safety profiles of conventional antimigraine drugs are compared with specific drugs that are exclusively used to block or antagonize the receptors. The safety profiles of all old drugs are also compared with recently marketed drugs that are used for the treatment of any form of migraine. The details are presented here.

\section{a) Non-steroidal anti-inflammatory drugs}

NSAIDS are known to have gastrointestinal side effects, including peptic ulcer, increased risk of myocardial infarction and heart failure. The incidence of side effects was proportional to dose [27].

\section{b) 5-hydroxy tryptamine (5HT)-agonists}

Triptans are known to have fewer side effects than ergot alkaloids. However, cardiovascular disease, which include uncontrolled hypertension is a contraindicated factor because triptans also vasoconstricts the coronary arteries [29].

\section{c) Ergots}

Clinical effect of ergots is due to their agonist activity primarily at 5$\mathrm{HT}^{1 \mathrm{~B} / \mathrm{D}}$ receptors and then $5-\mathrm{HT}^{1 \mathrm{~F}}$ receptors to a lesser extent [64]. This polypharmacology is believed to contribute to its adverse reactions. Side effects of ergots are reflected on their agonism on 5-HT1A receptors in which nausea and dysphoria are involved and at 5- $\mathrm{HT}^{2 \mathrm{~A}}$ receptors that leads to peripheral vasoconstriction. Side effects of ergots on cardiovascular activity is then related to its vasoconstrictive 
actions [32]. Ergots also act on dopamine D2 receptors, presenting nausea and vomiting in patients receiving this treatment [64]. Despite its inexpensiveness, ergots are associated with tolerability problems, potentials of vasoconstriction, poor bioavailability of its oral formulations, and risk of medication overuse, and its clinical use is relatively less extensive nowadays [33].

\section{d) Others drugs: (Botulinum toxin A)}

Most of the studies conducted have shown that Botulinum Toxin A is well tolerated by migraine sufferers, with patients exhibiting a significantly higher rate of treatment-related adverse effects when larger doses of BoNT/A are administered [48].

\section{Specific management}

\section{Calcitonin gene-related peptide antagonists}

\section{a) Fremanezumab}

In a study conducted by Dodick et al., at least one adverse event was reported by $66 \%$ of the participants who were injected with fremanezumab monthly at a higher dose compared to $8 \%$ who were given placebo. The adverse event profile of fremanezumab in this trial matches with previously conducted clinical trials, whereby no clinically significant patterns of serious adverse events are observed [49]. In another phase $2 \mathrm{~b}$, double-blind, placebo-controlled, parallelgroup study conducted by Bigal et al., adverse events were reported by $40 \%$ of patients in the placebo group, $53 \%$ of patients who received $675 / 225 \mathrm{mg}$ dose of fremanezumab and $47 \%$ of those who received $900 \mathrm{mg}$ fremanezumab. The most common adverse events experienced were mild injection-site pain and pruritus [50]. Cohen et al., conducted two randomized placebo-controlled studies on various subcutaneous doses of fremanezumab versus placebo as an add-ontherapy in episodic migraine and chronic migraine for a period of one month. Treatment-emergent adverse events were reported by $44 \%$ of patients who received placebo and $55 \%$ of patients receiving any other migraine preventive drug. Serious adverse events were recorded in only $2 \%$ of patients receiving fremanezumab. It can be concluded that fremanezumab is well tolerated with no severe treatment-related adverse events and deemed safe for use in migraine sufferers [51].

\section{b) Eptinezumab}

A group of 174 patients in the USA assigned to receive either treatment (ALD403) or placebo group. Among this, 57\% of the patients $(n=81)$ in the treatment group and $52 \%(n=43)$ in the placebo group experienced adverse events. The most frequent adverse events were upper respiratory tract infection (URTI), urinary tract infection (UTI), fatigue, back pain, arthralgia and nausea and vomiting. Six patients from the placebo group and 7 patients from the treatment group affected with URTI. There was an equal proportion of patients suffering from UTI and arthralgia, which was 4:1 in both placebo and treatment group. Moreover, there was an equal percentage of patients suffering from fatigue in both the treatment and placebo group. A 4:3 ratio of the patients was having back pain; 2 and $4 \%$ of the patients experienced nausea and vomiting after receiving placebo and ALD403, respectively. There were four serious adverse effects observed in the treatment group, while only one adverse effect was noted in the placebo group. Nonetheless, among both treatment and placebo group, there was no reported significant difference between laboratory safety data or vital signs. A-5.6 MMD were reported in the treatment group and-4.6 MMD for placebo group on the average change in day of migraine between baseline and weeks five to eight [52].

\section{c) Galcanezumab}

A study by Schuster et al., concluded that injection site reactions were more commonly observed in the group treated with galcanezumab than the control group. However, the treatment was well perceived without major adverse consequences [55]. In 2018, the safety and tolerability of galcanezumab were further investigated among 135 patients diagnosed with episodic or chronic migraine, and the findings revealed that most patients experienced treatment-emergent adverse effects (TEAE) include injection site reaction, nasopharyngitis, URTI, back pain and sinusitis [56].

\section{Calcitonin gene-related peptide receptor antagonists}

\section{a) Erenumab:}

A number of studies have been conducted appraising the safety profile of erenumab in the treatment of migraines. The ARISE [59] study with $70 \mathrm{mg}$ erenumab reported URTI, whereas LIBERTY [62] study showed injection-site pain in its $140 \mathrm{mg}$ intervention arm. Another study by Goadsby et al., [61] reported nasopharyngitis as its most frequent adverse event (AE) in both $70 \mathrm{mg}$ and $140 \mathrm{mg}$ erenumab treatment groups. In a phase 2 study by Tepper et al., [63] pain at the injection site was one of the most prominent AEs, occurring in $4 \%$ of each $70 \mathrm{mg}$ and $140 \mathrm{mg}$ erenumab groups, as well as muscle spasm in $4 \%$ of patients given with $140 \mathrm{mg}$ erenumab. Two serious AEs; traumatic orbital fracture and one incident of migraine attack were reported in the group treated with erenumab; however, it was assumed that both cases had nothing to do with the active drug [62]. Other common AEs include constipation [59, 61], nasopharyngitis [61-63], fatigue and sinusitis were also observed. As erenumab is of human IgG2 antibody, possibilities in the development of neutralizing anti-drug antibodies (ADA) decreases [65]. In ARISE, at week 12, 12 out of 279 patients treated with erenumab were shown to develop anti-erenumab-binding antibodies (AB). At week 4, 1 of the 12 patients showed positive neutralizing $A B, a$, however negative result for the same $A B$ in his subsequent visit [59]. Tepper et al., [63] also confirmed that occurrence of binding $\mathrm{AB}$ in $6 \%$ patients of $70 \mathrm{mg}$ group and $2 \%$ of $140 \mathrm{mg}$ group, however, without neutralising $\mathrm{AB}$. There was no relationship between this occurrence and $\mathrm{AE}$ in this study [63]. Incidence of anti-erenumab $\mathrm{AB}$ is rare and remit in most of studies. Apart from serum chemistry, no notable abnormalities and alterations were reported associated to primary vital signs, electrocardiogram (ECG) testing, and laboratory monitoring in all patients participating in all studies evaluated above [59, 63-65]. In a study, only one patient showed abnormal rise in alanine and aspartate aminotransferase at week 4 of study; the reading then returned to baseline in subsequent visit in week 8 [63]. As erenumab does not undergo hepatic metabolism, there were no significant impacts on liver enzymes, unlike the hepatotoxicity associated in treatment with telcagepant, a small molecule CGRP receptor antagonist [66, 67]. There were no deaths reported in studies conducted for erenumab $[59,61,62]$. The incidence of AEs in both erenumab and placebo interventions were similar [59, 61-65] and this further confirms the safety of the administration of erenumab. Erenumab is preferred as migraine preventive treatment with positive efficacy and safety profile, contributed by its pharmacokinetics [38].

\section{CONCLUSION}

All the existing antimigraine therapies were included for comparison of efficacy and safety in controlling repetitive migraine attack. Upon comparison, there are four migraine prevention drugs were considered more effective in terms of controlling the severity and frequency of migraine attack; there are Fremanezumab, Eptinezumab, Galcanezumab and Erenumab. Among these, Erenumab, a CGRP receptor antagonists at a dose of 70 and $140 \mathrm{mg}$ was found to be most effective in controlling the frequency of migraine episodes. Erenumab may be a suitable alternative therapeutic agent in EM patients whom other traditional preventive treatments are contraindicated, unsuccessful or poorly tolerated as it produces more than 50 percent reduction in mean number of MMD in just few weeks of therapy.

\section{ACKNOWLEDGMENT}

The authors would like to thank the management and staffs of International Medical University for the facilities provided to carry out the review.

\section{FUNDING}

Nil

\section{AUTHORS CONTRIBUTIONS}

All the authors have contributed equally. 


\section{CONFLICT OF INTERESTS}

Declared none

\section{REFERENCES}

1. Bartleson JD, Cutrer FM. Migraine update-diagnosis and treatment. Minn Med 2010;93:36-41.

2. Goadsby PJ, Holland PR, Martins-Oliveira M, Hoffmann J, Schankin C, Akerman S. Pathophysiology of migraine: a disorder of sensory processing. Physiol Rev 2017;97:553-622.

3. Buse DC, Scher AI, Dodick DW, Reed ML, Fanning KM, Manack Adams A, et al. Impact of migraine on the family: perspectives of people with migraine and their spouse/domestic partner in the CaMEO study. Mayo Clin Proc 2016;91:596-611.

4. Ferrari MD, Klever RR, Terwindt GM, Ayata C, van den Maagdenberg AM. Migraine pathophysiology: lessons from mouse models and human genetics. Lancet Neurol 2015;14:65-80.

5. Goadsby PJ, Lipton RB, Ferrari MD. Migraine-current understanding and treatment. N Engl J Med 2002;346:257-70.

6. Lipton RB, Bigal ME, Diamond M, Freitag F, Reed ML, Stewart WF. AMPP Advisory Group. Migraine prevalence, disease burden, and the need for preventive therapy. Neurology 2007;68:343-9.

7. Steiner TJ, Stovner LJ, Vos T. GBD 2015: migraine is the third cause of disability in under 50s. J Headache Pain 2016;17:104.

8. Lipton RB, Stewart WF, Diamond S, Diamond ML, Reed M. Prevalence and burden of migraine in the United States: data from the American Migraine Study II. Headache 2001;41:646-57.

9. Stewart WF, Shechter A, Rasmussen BK. Migraine prevalence. A review of population-based studies. Neurology 1994;44:S17-23.

10. Robbins MS, Lipton RB. The epidemiology of primary headache disorders. Semin Neurol 2010;30:107-9.

11. Menon R. Women more prone to headaches: study. Deccan Herald. Available from: https://www.deccanherald.com/content/156167/womenmore-prone-headaches-study.html. [Last accessed on 10 Feb 2020]

12. Steiner TJ, Stovner LJ, Birbeck GL. Migraine: the seventh disabler. Cephalalgia 2013;33:289-90.

13. Steiner TJ, Stovner LJ, Birbeck GL. Migraine: the seventh disabler. Headache 2013;53:227-9.

14. Steiner TJ, Stovner LJ, Birbeck GL. Migraine: the seventh disabler. J Headache Pain 2013;14:1.

15. Steiner TJ, Birbeck GL, Jensen RH, Katsarava Z, Stovner LJ, Martelletti P. Headache disorders are the third cause of disability worldwide. J Headache Pain 2015;16:58.

16. GBD 2013 Risk Factors Collaborators, Forouzanfar MH, Alexander L, Anderson HR, Bachman VF, Biryukov S, et al. Global, regional, and national comparative risk assessment of 79 behavioral, environmental and occupational, and metabolic risks or clusters of risks in 188 countries, 1990-2013: a systematic analysis for the Global Burden of Disease Study 2013. Lancet 2015;386:2287-323.

17. GBD 2015 Disease and Injury Incidence and Prevalence Collaborators. Global, regional, and national incidence, prevalence, and years lived with disability for 310 diseases and injuries, 1990-2015:a systematic analysis for the Global Burden of Disease Study 2015. Lancet 2016;388:1545-602.

18. GBD 2016 Disease and Injury Incidence and Prevalence Collaborators. Global, regional, and national incidence, prevalence, and years lived with disability for 328 diseases and injuries for 195 countries, 1990-2016: a systematic analysis for the Global Burden of Disease Study 2016. Lancet 2017;390:1211-59.

19. Chan KY, Vermeersch S, de Hoon J, Villalon CM, Maassenvandenbrink A. Potential mechanisms of prospective antimigraine drugs: a focus on vascular (side) effects. Pharmacol Ther 2011;129:332-51.

20. Charles A. The evolution of a migraine attack-a review of recent evidence. Headache 2013;53:413-9.

21. Burch R, Rizzoli P, Loder E. The prevalence and impact of migraine and severe headache in the United States: fig. and trends from government health studies. Headache 2018;58:496-505.
22. Deen M, Correnti E, Kamm K, Kelderman T, Papetti L, RubioBeltran E, et al. Blocking CGRP in migraine patients-a review of pros and cons. J Headache Pain. 2017;18:96.

23. Russo AF. Calcitonin gene-related peptide (CGRP): a new target for migraine. Annu Rev Pharmacol Toxicol 2015;55:533-52.

24. Uddman R, Edvinsson L, Ekman R, Kingman T, McCulloch J. Innervation of the feline cerebral vasculature by nerve fibers containing calcitonin gene-related peptide: trigeminal origin and co-existence with substance P. Neurosci Lett 1985;62:131-6.

25. Keller JT, Marfurt CF. Peptidergic and serotoninergic innervation of the rat dura mater. J Comp Neurol 1991;309:515-34.

26. Yeh J, Akinci A, Al Shaker M, Chang MH, Danilov A, Guillen R, et al. Monoclonal antibodies for chronic pain: a practical review of mechanisms and clinical applications. Mol Pain 2017;13:174480691774023.

27. Pardutz A, Schoenen J. NSAIDs in the acute treatment of migraine: a review of clinical and experimental data. Pharmaceuticals (Basel) 2010;3:1966-87.

28. Mekaj YH, Daci FT, Mekaj AY. New insights into the mechanisms of action of aspirin and its use in the prevention and treatment of arterial and venous thromboembolism. Ther Clin Risk Manag 2015;11:1449-56.

29. Walter S, Alibhoy A, Escandon R, Bigal ME. Evaluation of cardiovascular parameters in cynomolgus monkeys following IV administration of LBR-101, a monoclonal antibody against calcitonin gene-related peptide. MAbs 2014;6:871-8.

30. Lovati C, Giani L, Mariotti D, Alessandro C, Tabaee Damavandi $\mathrm{P}$, Mariani C, et al. May migraine attack response to triptans be a predictor of the efficacy of Onabotulinum toxin-a prophylaxis? Neurol Sci 2018;39:153-4.

31. Peck KR, Johnson YL, Smitherman TA. Migraine. Handb Clin Neurol 2016;138:283-93.

32. Bell IM. Calcitonin gene-related peptide receptor antagonists: new therapeutic agents for migraine. J Med Chem 2014;57:7838-58.

33. Monteith TS, Goadsby PJ. Acute migraine therapy: new drugs and new approaches. Curr Treat Options Neurol 2010;13:1-14.

34. Silberstein S, Mathew N, Saper J, Jenkins S. Botulinum toxin type a as a migraine preventive treatment. For the BOTOX migraine clinical research group. Headache 2000;40:445-50.

35. Melo Carrillo A, Strassman AM, Nir RR, Schain AJ, Noseda R, Stratton J, et al. Fremanezumab-a humanized monoclonal antiCGRP antibody-inhibits thinly myelinated (AS) but not unmyelinated (C) meningeal nociceptors. I Neurosci 2017;37:10587-96.

36. Schwedt TJ. Chronic migraine. Br Med J 2014;348:g1416.

37. Monteith D, Collins EC, Vandermeulen C, Van Hecken A, Raddad E, Scherer JC, et al. Safety, tolerability, pharmacokinetics, and pharmacodynamics of the CGRP binding monoclonal antibody LY2951742 (Galcanezumab) in healthy volunteers. Front Pharmacol. 2017;8:740.

38. Bloudek LM, Stokes M, Buse DC, Wilcox TK, Lipton RB, Goadsby $\mathrm{PJ}$, et al. Cost of healthcare for patients with migraine in five European countries: results from the International Burden of Migraine Study (IBMS). J Headache Pain 2012;13:361-78.

39. Shi L, Lehto SG, Zhu DX, Sun H, Zhang J, Smith BP, et al. Pharmacologic characterization of AMG 334, a potent and selective human monoclonal antibody against the calcitonin gene-related peptide receptor. J Pharmacol Exp Ther 2016;356:223-31.

40. Hostetler ED, Joshi AD, Sanabria Bohorquez S, Fan H, Zeng Z, Purcell M, et al. In vivo quantification of calcitonin gene-related peptide receptor occupancy by telcagepant in rhesus monkey and human brain using the positron emission tomography tracer ${ }^{11}$ CMK-4232. J Pharmacol Exp Ther 2013;347:478-86.

41. Eftekhari S, Salvatore C, Johansson S, Chen TB, Zeng Z, Edvinsson L. Localization of CGRP, CGRP receptor, PACAP and glutamate in the trigeminal ganglion. Relation to the bloodbrain barrier. Brain Res 2015;1600:93-109.

42. Vu T, Ma P, Chen JS, de Hoon J, Van Hecken A, Yan L, et al. Pharmacokinetic-pharmacodynamic relationship of erenumab (AMG 334) and capsaicin-induced dermal blood flow in healthy and migraine subjects. Pharm Res 2017;34:1784-95. 
43. Hougaard A, Tfelt Hansen P. Review of dose-response curves for acute antimigraine drugs: triptans, 5-HT1F agonists and CGRP antagonists. Expert Opin Drug Metab Toxicol 2015;11:1409-18.

44. Schuster NM, Vollbracht S, Rapoport AM. Emerging treatments for primary headache disorders. Neurol Sci 2015;36:109-13.

45. De Hoon JN, Poppe KA, Thijssen HH, Struijker Boudier HA, Van Bortel LM. Dihydroergotamine: discrepancy between arterial, arteriolar and pharmacokinetic data. Br J Clin Pharmacol 2001;52:45-51.

46. Winner P, Dalessio D, Mathew N, Sadowsky C, Turkewitz L, Sheftell F, et al. Office-based treatment of acute migraine with dihydroergotamine mesylate. Headache 1993;33:471-5.

47. Evers S, Gralow I, Bauer B, Suhr B, Buchheister A, Husstedt I, et al. Sumatriptan and ergotamine overuse and drug-induced headache: a clinicoepidemiologic study. Clin Neuropharmacol 1999;22:201-6.

48. Freitag FG. Importance of botulinum toxin for the prevention of migraine. Expert Rev Neurother 2010;10:339-40.

49. Dodick WD, Silberstein DS, Bigal ME, Goadsby PJ, Blankenbiller T, Grozinski-Wolff M, et al. Effect of fremanezumab compared with placebo for prevention of episodic migraine-a randomized clinical trial. JAMA 2018;319:1999-2008.

50. Bigal ME, Edvinsson L, Rapoport AM, Lipton RB, Spierings EL, Diener HC, et al. Safety, tolerability, and efficacy of TEV-48125 for preventive treatment of chronic migraine: a multicentre, randomised, double-blind, placebo-controlled, phase $2 \mathrm{~b}$ study. Lancet Neurol 2015;14:1091-100.

51. Cohen JM, Dodick DW, Yang R, Newman LC, Li T, Aycardi E, et al. Fremanezumab as an add-on treatment for patients treated with other migraine preventive medicines. Headache 2017;57:1375-84

52. Dodick DW, Goadsby PJ, Silberstein SD, Lipton RB, Olesen J, Ashina $\mathrm{M}$, et al. Safety and efficacy of ALD403, an antibody to calcitonin gene-related peptide, for the prevention of frequent episodic migraine: a randomized, double-blind, placebo-controlled, exploratory phase 2 trial. Lancet Neurol 2014;13:1100-7.

53. Dodick DW, Lipton RB, Silberstein S, Goadsby PJ, Biondi D, Hirman J, et al. Eptinezumab for prevention of chronic migraine: a randomized phase $2 \mathrm{~b}$ clinical trial. Cephalalgia 2019;39:1075-85.

54. Pellesi L, Guerzoni S, Pini LA. Spotlight on anti-CGRP monoclonal antibodies in migraine: the clinical evidence to date. Clin Pharmacol Drug Dev 2017;6:534-47.

55. Schuster NM, Rapoport AM. New strategies for the treatment and prevention of primary headache disorders. Nat Rev Neurol 2016;12:635-50.

56. Camporeale A, Kudrow D, Sides R, Wang S, Van Dycke A, Selzler $\mathrm{KJ}$, et al. A phase 3, long-term, open-label safety study of
Galcanezumab in patients with migraine. BMC Neurol 2018;18:188.

57. Skljarevski V, Matharu M, Millen BA, Ossipov MH, Kim BK, Yang JY. Efficacy and safety of galcanezumab for the prevention of episodic migraine: results of the EVOLVE-2 Phase 3 randomized controlled clinical trial. Cephalalgia 2018;38:1442-54.

58. Forderreuther S, Zhang Q, Stauffer VL, Aurora SK, Lainez MJA. Preventive effects of galcanezumab in adult patients with episodic or chronic migraine are persistent: data from the phase 3 , randomized, double-blind, placebo-controlled evolve-1, evolve- 2 , and regain studies. J Headache Pain 2018;19:121.

59. Dodick DW, Ashina M, Brandes JL, Kudrow D, Lanteri Minet M, Osipova V, et al. ARISE: a phase 3 randomized trial of erenumab for episodic migraine. Cephalalgia 2018;38:1026-37.

60. Sun H, Dodick DW, Silberstein S, Goadsby PJ, Reuter U, Ashina $\mathrm{M}$, et al. Safety and efficacy of AMG 334 for prevention of episodic migraine: a randomised, double-blind, placebocontrolled, phase 2 trial. Lancet Neurol 2016;15:382-90.

61. Goadsby PJ, Reuter U, Hallstrom Y, Broessner G, Bonner JH, Zhang $\mathrm{F}$, et al. A controlled trial of erenumab for episodic migraine. N Engl J Med 2017;377:2123-32.

62. Reuter U, Goadsby PJ, Lanteri Minet M, Wen S, Hours Zesiger P, Ferrari M, et al. Efficacy and tolerability of erenumab in patients with episodic migraine in whom two-to-four previous preventive treatments were unsuccessful: a randomised, double-blind, placebo-controlled, phase $3 \mathrm{~b}$ study. Lancet 2018;392:2280-7.

63. Tepper S, Ashina M, Reuter U, Brandes JL, Dolezil D, Silberstein S, et al. Safety and efficacy of erenumab for preventive treatment of chronic migraine: a randomized, double-blind, placebo-controlled phase 2 trial. Lancet Neurol 2017;16:425-34.

64. Silberstein SD, McCrory DC. Ergotamine and dihydroergotamine: history, pharmacology, and efficacy. Headache 2003;43:144-66.

65. Levin M, Silberstein SD, Gilbert R, Lucas S, Munsie L, Garrelts A, et al. Basic considerations for the use of monoclonal antibodies in migraine. Headache 2018;58:1689-96.

66. Ho TW, Connor KM, Zhang Y, Pearlman E, Koppenhaver J, Fan X, et al. Randomized controlled trial of the CGRP receptor antagonist telcagepant for migraine prevention. Neurology 2014;83:958-66.

67. Aimovig (erenumab-aooe) injection (package insert). Thousand Oaks, CA: Amgen Inc; 2018.

68. Skljarevski V, Oakes TM, Zhang Q, Ferguson MB, Martinez J, Camporeale A, et al. Effect of different doses of galcanezumab vs placebo for episodic migraine prevention: a randomized clinical trial. JAMA Neurol 2018;75:187-93. 